Fi nger-att achment devi ce for the feedback of gri pping and pul I ing force in a mani pul at ing system for brai $\mathrm{n}$ tumor resecti on

\begin{tabular}{|c|c|}
\hline 著者 & $\begin{array}{l}\text { Chi nbe H r oyuki, Yoneyana Takeshi, Wat anabe } \\
\text { Tet suyou, M yashi t a Kat suyoshi, Nakada } \\
\text { M t sut oshi }\end{array}$ \\
\hline 著者別表示 & 米山 猛, 渡辺 哲陽, 宮下 勝吉, 中田 光俊 \\
\hline $\begin{array}{l}\text { j our nal or } \\
\text { publ i cat i on title }\end{array}$ & $\begin{array}{l}\text { I nt er nat i onal J our nal of Comput er Assi st ed } \\
\text { Radi ol ogy and Surgery }\end{array}$ \\
\hline vol une & 13 \\
\hline nunber & 1 \\
\hline page $r$ ange & 312 \\
\hline year & 2018-01- 01 \\
\hline URL & ht t p: //doi . or g/10. 24517/00042068 \\
\hline
\end{tabular}




\title{
Finger-attachment device for the feedback of gripping and pulling force in a manipulating system for brain tumor resection
}

\author{
Hiroyuki Chinbe, Takeshi Yoneyama, Tetsuyou Watanabe \\ School of Mechanical Engineering, Kanazawa University \\ Kakuma-machi, Kanazawa 920-1192, Japan \\ 81-76-234-4683 \\ yoneyama@se.kanazawa-u.ac.jp
}

Katsuyoshi Miyashita, Mitsutoshi Nakada

Department of Neurosurgery Graduate School of Medical Science, Kanazawa University

Takara-machi, Kanazawa 920-8641, Japan

\begin{abstract}
Purpose: Development and evaluation of an effective attachment device for a bilateral brain tumor resection robotic surgery system based on the sensory performance of the human index finger in order to precisely detect gripping- and pulling-force feedback.

Methods: First, a basic test was conducted to investigate the performance of the human index finger in the gripping- and pulling-force feedback system. Based on the test result, a new fingerattachment device was designed and constructed. Then, discrimination tests were conducted to assess the pulling force and the feedback on the hardness of the gripped material.
\end{abstract}

Results: The results of the basic test show the application of pulling force on the side surface of the finger has an advantage to distinguish the pulling force when the gripping force is applied on the finger-touching surface. Based on this result, a finger-attachment device that applies a gripping force on the finger surface and pulling force on the side surface of the finger was developed. By conducting a discrimination test to assess the hardness of the gripped material, an operator can distinguish whether the gripped material is harder or softer than a normal brain tissue. This will help in confirming whether the gripped material is a tumor. By conducting a discrimination test to assess the pulling force, an operator can distinguish the pulling-force resistance when attempting to pull-off the soft material. Pulling-force feedback may help avoid the breaking of blood pipes when they are trapped in the gripper or attached to the gripped tissue.

Conclusion: The finger-attachment device that was developed for detecting gripping- and pullingforce feedback may play an important role in the development of future neurosurgery robotic systems for precise and safe resection of brain tumors.

Keywords 
Neurosurgery, robotic surgery, brain tumor, manipulator, force feedback 


\section{Introduction}

In recent years, the use of manipulators with endoscopes in operations has become popular owing to their minimal invasiveness [1-2]. Master-slave surgical robotics has also been widely used in the field of abdominal and urological surgeries owing to its precise performance in surgeries [3-6]. However, robotic surgery systems have not been used in brain tumor resection because there is only a narrow space available to insert a manipulator and it is necessary to perform extremely delicate surgery for the resection of a precise area of a tumor without causing any damage to the surrounding normal tissues and blood pipes [7-8].

Some studies have investigated surgical robotics for neurosurgery. Sutherland et al. developed a manipulating system named "neuroArm," which has two microsurgical tools, namely bipolar forceps and suction [9-10]. Goto et al. developed "NeuRobot," which has three robot arms and an endoscope [11-12]. Mitsuishi et al. developed a manipulating system that can monitor micro blood pipes in the brain, and they succeeded in joining an artificial micro blood pipe with a 0.3-mm diameter with another one [13-14]. In addition, Arata et al. have been developing volume control suction tools with flexible manipulators to remove brain tumors [15-16].

However, these manipulator systems do not have a force feedback system, which is used to find the mechanical compliance of tissues undergoing the microsurgery robotic operation [17-21]. The authors developed a force-detecting gripper and a force feedback system for neurosurgery applications [22]. Manipulation of handle and finger attachments can be improved by applying gripping and pulling forces on the operator's finger [23]. Thus far, the gripping force applied on a soft material that has the same hardness as that of a brain tissue could be transferred to the operating handle so that the operator could feel the gripping force at his/her finger. However, in terms of the pulling-force feedback from the gripped material, the operator could not feel the pulling force or resistance completely when he gripped the material rather tightly. This may be due to the fact that under a tight grip, the operator may feel greater pressure on the finger surface.

Based on these results, in this study, a basic experiment on how to transfer the pulling force to the operator's finger was conducted; then, a new finger-attachment device with good performance in terms of pulling-force feedback was developed and its availability was examined.

\section{Basic experiment for analyzing pulling-force feedback}

An experiment was conducted using a simple device to investigate how the finger feels a pulling force when a gripping force is applied to it and to determine the type of attachment that would be better for obtaining pulling-force feedback. Figure 1 shows the devices and methods used to apply gripping and pulling forces. Attachment $\mathrm{A}$ is a device that holds the index finger from between its face and the rear surface; it applies a gripping force on the plate that contacts the finger face and applies a pulling force on the finger through the same plate. Attachment B is a device that holds the finger from between its side surfaces. It applies a gripping force on the finger face through a thin plate, which is not connected to the attachment, and applies a pulling force through the attachment independently.

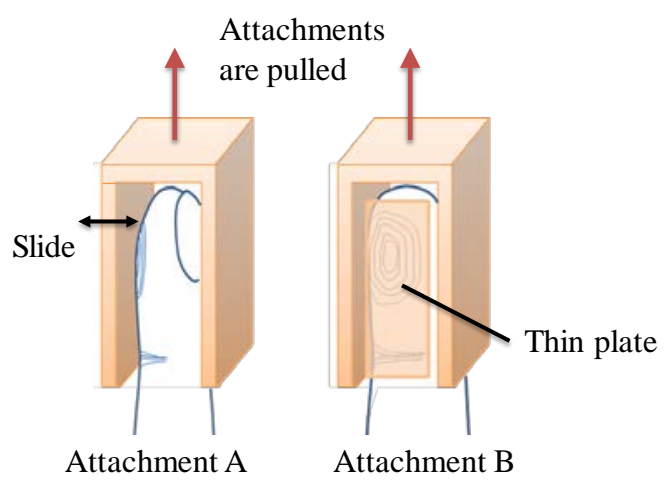

Fig. 1 Experimental devices. 
First, a test was conducted to distinguish the absolute pulling force with four patterns using eight operators of seven males and one female aged from 21 to 23 years with no medical background. They were all right-handed, and none of them reported any difficulties regarding their hands and haptics. Figure 2 illustrates the experimental method. An operator held the grip pole with his/her thumb and three fingers (the middle, ring, and little fingers). The operator fit the attachment on the index finger and applied a gripping force on the force gauge using the finger face. The gripping force applied by the subject was $3 \mathrm{~N}$, which was displayed by the force gauge. Then, the operator closed his eyes and applied a pulling force on the finger attachment using a wire; this time, the pulling force applied was detected using another sensor. In this test, after the operator applied the gripping force, the pulling force increased with a rate of $0.1 \mathrm{~N} / \mathrm{s}$, and when the operator felt a pulling force, he/she touched the pushing button using his/her another hand and the pulling force was recorded automatically. As can be seen in Fig. 2, the first pattern shows a gripping force of $3 \mathrm{~N}$ with Attachment $\mathrm{A}$, the second pattern shows no gripping force with Attachment $\mathrm{A}$, the third pattern shows a gripping force of $3 \mathrm{~N}$ with Attachment $\mathrm{B}$, and the final pattern shows no gripping force with Attachment B. The test was conducted three times, and the average value was obtained. This test determined the minimum pulling force that an operator can distinguish. The reason why a gripping force of $3 \mathrm{~N}$ was applied on the robotic surgery system is that when the gripping force applied by the slave manipulator on the soft material, whose hardness was the same as that of brain tissue, was approximately $0.7 \mathrm{~N}$, the gripping force feedback on the master finger attachment was optimal, i.e., approximately $3 \mathrm{~N}$, for the operator.

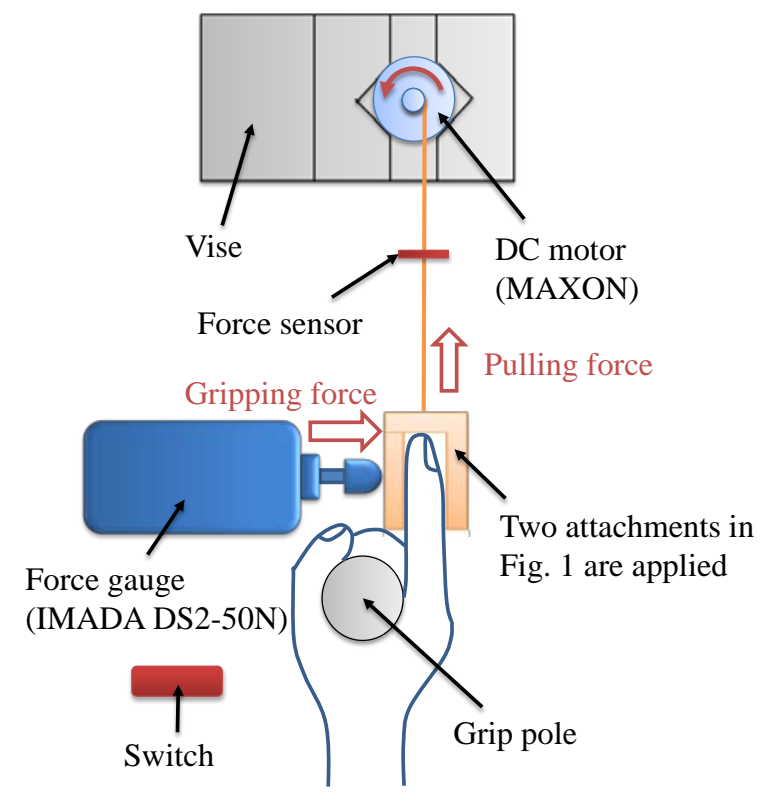

Fig. 2. Schematic of the experimental method.

Next, another test was performed to distinguish the change in the pulling force. In this test, after the operator applied a gripping force, some part of the initial pulling force was applied for $5 \mathrm{~s}$ and then the pulling force changed to some extent. The operator answered whether the pulling force increased or decreased. The result was recorded (correct or incorrect, based on the answer given by the subject). When the operator answered that he could not distinguish between an increase and a decrease, the result was recorded as incorrect. The change in the magnitude of the pulling force ranged from a large value to a small value with a $0.1-\mathrm{N}$ step. The minimum value of the pulling force was changed so that the rate of obtaining correct answers would be $80 \%$ or higher, which was decided as the minimum value to distinguish the pulling-force change. The initial pulling forces were set at 1 and $2 \mathrm{~N}$. The same four patterns were also tested by the same eight operators.

\section{Results of the basic test}

Figure 3 shows the results of the absolute pulling force distinguished with standard deviation. In each attachment, the threshold of the pulling force increases upon the application of a gripping force. When no gripping force is applied, the threshold value with Attachment B is higher than that with Attachment A; however, when a gripping force is applied, the threshold value with 
Attachment B is lower. This result shows that the sensitivity of the pulling force decreases upon the application of the gripping force and that when a pulling force is applied on the side surface of the finger, the sensitivity is better than that in the case in which the pulling force is applied on the same finger surface with the gripping force. These results also show that even when a gripping force of $3 \mathrm{~N}$ is applied, a pulling force $>1 \mathrm{~N}$ can be distinguished.

Figure 4 shows the results obtained from the second test performed to detect the change in the pulling force. In each pattern, the left and right bars show the results obtained with the application of an initial pulling force of 1 and $2 \mathrm{~N}$, respectively. In addition, in each pattern, the difference threshold increases with the pulling force of $2 \mathrm{~N}$, as explained by Weber-Fechner's law [24]; moreover, the threshold increases upon the application of a gripping force. Therefore, the sensitivity of the change in the pulling force decreases due to the initial pulling force and application of the gripping force. Although, in the case of no gripping force, the sensitivity of the change in the pulling force with Attachment B was lower than that with Attachment A, it remained unchanged under the application of a gripping-force.

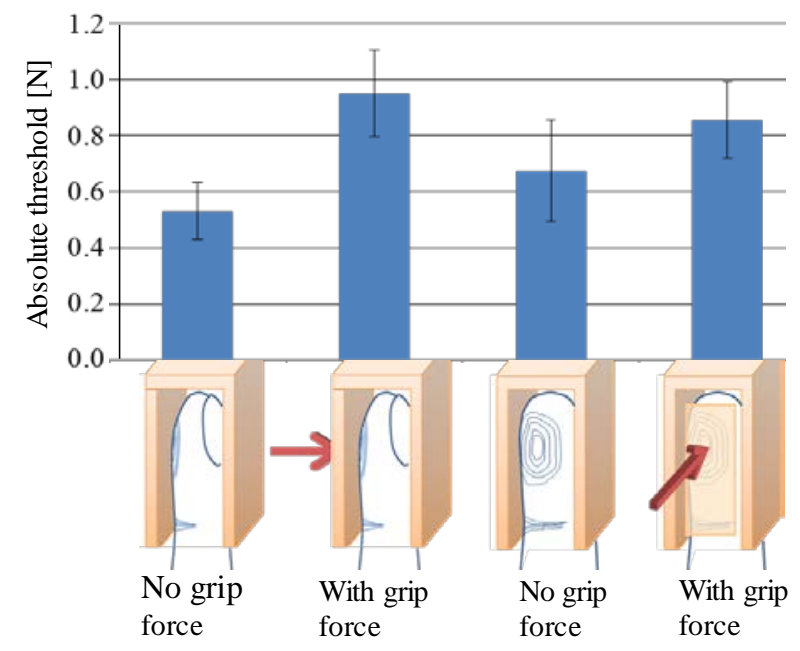

Attachment A

Attachment B

Fig. 3. Absolute threshold of the pulling force.

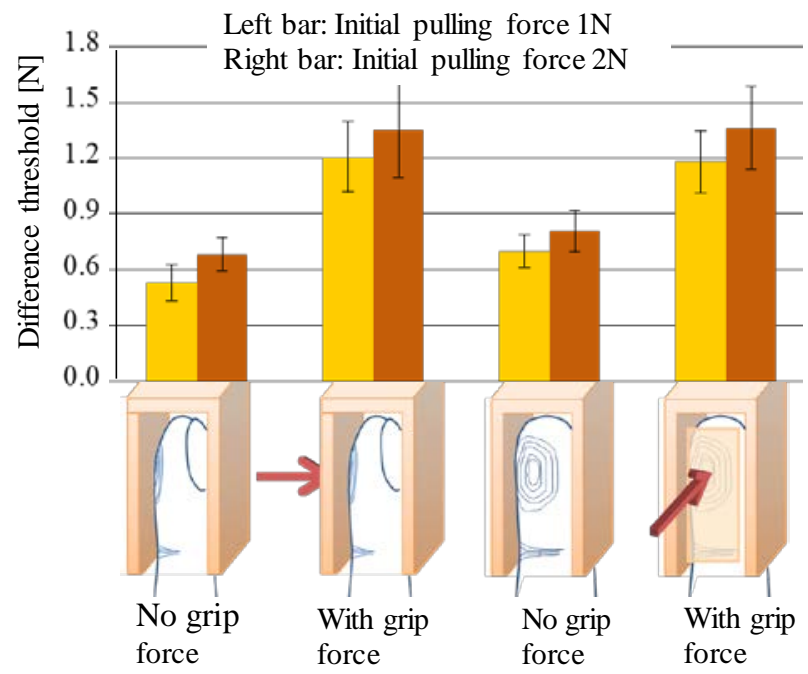

Attachment A

Attachment B

Fig. 4. Difference threshold of the pulling force.

Figure 5 summarizes the sensitivity of the pulling force based on Figs. 3 and 4 . In the case of no gripping force, the difference threshold for the pulling force with Attachment $\mathrm{A}$ is lower than with Attachment B. However, when a gripping force is applied, the difference threshold of the 
pulling force with Attachment B is slightly lower than or same as that with Attachment A. In brain tumor resection, the operator holds the tumor using the manipulator with the force feedback of the gripping force. In this case, the feedback of the pulling force on the side surface of the finger will be better when using Attachment B.

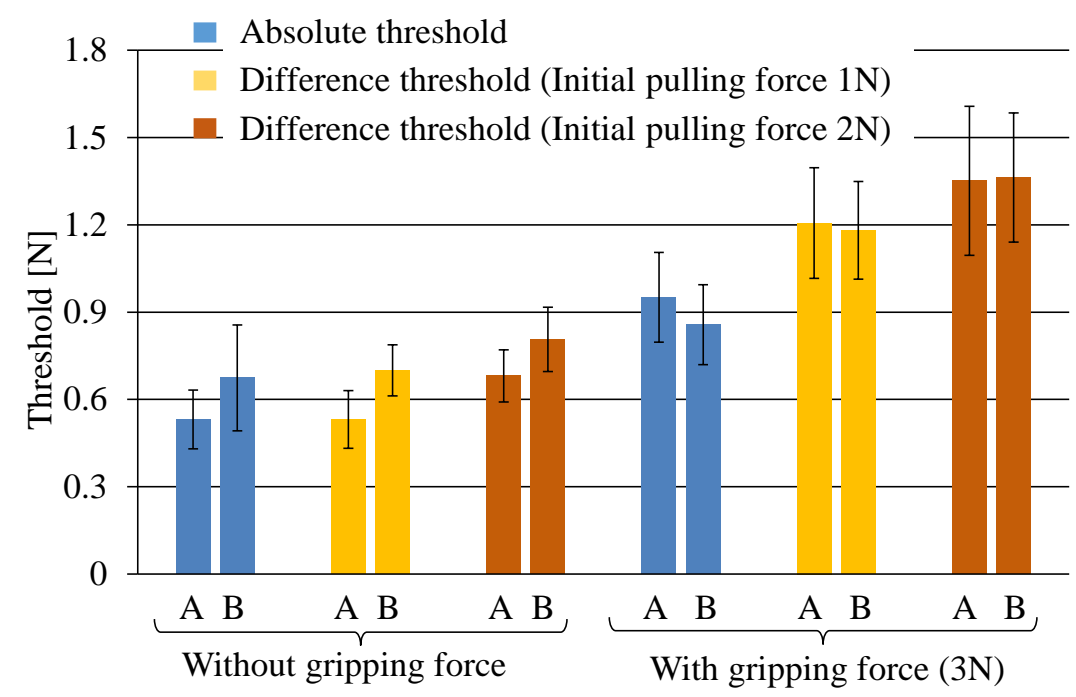

Fig. 5. Difference in pulling-force application. The left bars show the results for Attachment A, and the right bars show the results for Attachment B.

\section{Design and construction of the finger-attachment device}

Based on the results of the basic experiments, a new finger-attachment device was designed and constructed to be placed on the master operating handle in the manipulator system.

The functional requirements for the new device were as follows:

(a) Applying gripping force on the index finger surface and independently applying pulling force on the side surface of the finger.

(b) Each applied force is detected by a sensor and the force should be controllable.

(c) The attachment should be able to function with different index-finger sizes.

Figure 6 illustrates the constructed device, which comprises a holding pole. The finger attachment was connected with the lever that rotates around the axis near the pole. An operator held the pole with his/her thumb and three fingers (the middle, ring, and little fingers). The index finger was set in the finger attachment. To close the gripper at the slave manipulator, the operator closed the index finger by pushing the attachment through the finger surface. The pushing force was detected as gripping force by a force sensor that was installed between the lever and the finger attachment. The gripping force was controlled to some magnified gripping force in the slave manipulator by a servomotor installed in the center pole as the lever pushing resistance through the driving belt between the motor and the lever. On the other hand, the finger attachment can move in the front and back directions in the rotating lever. The front and back motion was driven by a ball screw motion using another servomotor. Through this motion, the pulling force was applied on the index finger, detected using a force sensor attached to the slide block in the ball screw mechanism, and controlled by the driving motor of the ball screw. To enable the finger attachment to function with different finger sizes, a setting plate was used to fit the side surface of the finger in the attachment. This plate can be shifted and fixed by using a set screw. The finger is folded by using silicon rubber in the holder to prevent the finger from slipping in the attachment.

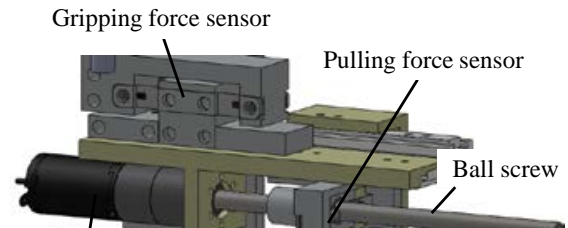


Fig. 6. Constructed device.

Control system

Figure 7 shows the master-slave operating system used in this study. The slave manipulator moves according to the master manipulator's movement, and the acting force on the slave manipulator is transferred to the master operation handle. As the detail of the control system is described in Refs. [23] and [23], only the basic system is described here.

For gripping-force feedback, the operation output $V_{\text {out }}$ from the computer to the servomotor can be written as

$$
V_{\text {out }}=K_{f}\left(S_{f} f_{s}-f_{m}\right)-C_{m} V_{m}
$$

where $K_{f}$ is the gain of the force, $S_{f}$ is the magnification factor of the force, $f_{s}$ is the force in the slave manipulator, $f_{m}$ is the force in the master manipulator, $C_{m}$ is the gain of the damper, and $V_{m}$ is the rotation rate of the lever. In this system, the force detected at the slave manipulator was magnified $K_{f}$ times the gripping force at the master manipulator. When there is no gripping force at the slave manipulator, the servomotor in the master manipulator assists the master gripping motion; $C_{m} V_{m}$ is a cushion to transfer the force moderately even when the impact force is applied on the slave gripper.

For pulling-force feedback, the operation output $V_{\text {out }}$ from the computer can be written as

$$
V_{\text {out }}=K_{s} \sin (20 \pi t)+K_{f}\left(S_{f} f_{s}-f_{m}\right)-C_{m} V_{m},
$$

where $K_{s}$ is the amplitude of the sine function and $t$ is the time. The first element in Equation (2) is to keep the motor torque when the detecting force at the slave was too small to move the device unit against the static friction on the ball screw in the finger devise.

The control program was written in $\mathrm{C}++$, and real-time feedback was implemented. When the operator moves the master manipulator, the slave manipulator moves without delay. Each part of the slave manipulator is controlled by the motion positions or angles in the master manipulator. Thus, each motion in the slave manipulator corresponds to a motion in the master manipulator.

The diameter of the top gripper in the slave manipulator was $3 \mathrm{~mm}$, and the manipulator had five degrees of freedom [25]. Both the gripping and pulling forces at the slave gripper were detected independently by strain gauges fit at the gripping element, as shown in Fig. 8.




Fig. 7. Entire master-slave system.

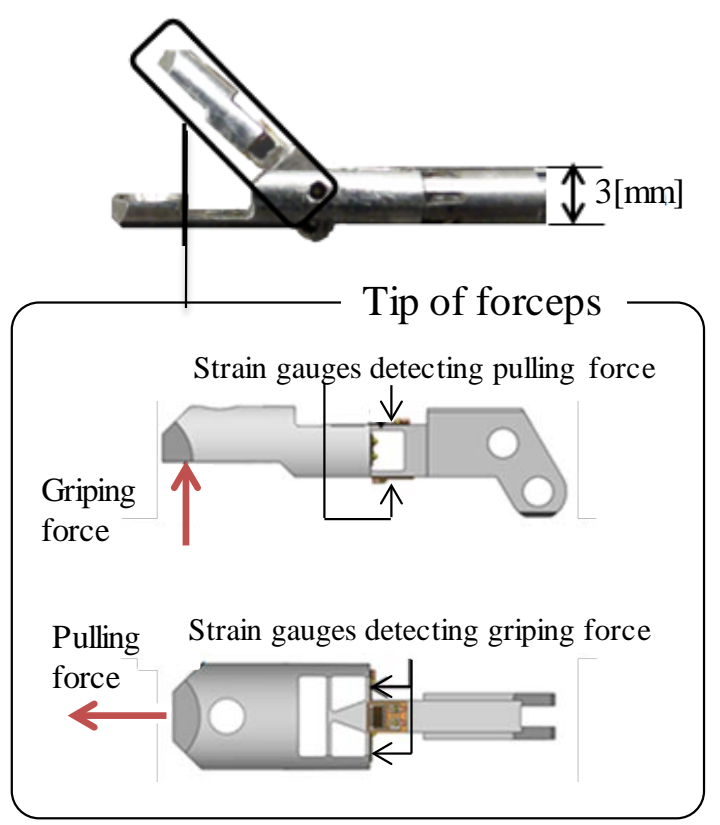

Fig.8. Force-detecting gripper at the tip of the forceps.

\section{Experiment to obtain the distinguished hardness of the gripped material}

By using the master-slave manipulation system with the newly developed finger attachment, an examination was conducted to distinguish the difference in the hardness of the gripped material. The Young's modulus of the brain tissue was reported to range from 10 to $50 \mathrm{kPa}$ [26], and the shearing elasticity of the brain tissue was reported to be $5 \mathrm{kPa}$ [27]. Because the Young's modulus was three times larger than the shearing elasticity for the biomaterial, youung's modulus of the brain tissue can be estimated as $\sim 15 \mathrm{kPa}$. The hardness of the brain tumor differed according to the tumor position and the type of tumor. According to M. Imbault, differences in the Young's moduli of meningioma, low-grade gliomas, high-grade gliomas, and metastasis when compared to those of normal brain tissue were 25.8, 16.4, 4.1, and $9.4 \mathrm{kPa}$, respectively [28]. On the other hand, the elastic modulus of pituitary macroadenomas was $\sim 5 \mathrm{kPa}$, which indicates that it is softer than normal brain tissue [29].

Based on these facts, seven types of gelatin with different hardness values close to that of the brain tissue and with different Young's moduli were prepared by changing the amount of gelatin powder in hot water during the production process (Table 1). 


\begin{tabular}{c|c}
\hline \hline Young's modulus [kPa] & Difference from $15 \mathrm{kPa}$ \\
\hline 6.7 & -8.3 \\
8.7 & -6.3 \\
11.4 & -3.6 \\
15.0 & 0.0 \\
19.7 & 4.7 \\
25.8 & 10.8 \\
33.8 & 8.8 \\
\hline \hline
\end{tabular}

Experimental procedure

The experiment was performed with nine right-handed operators of five males and four females aged 20-25 years; they had no medical background. None of them reported any difficulties regarding their hands and haptics. First, an operator gripped the gelatin with a 15-kPa Yong's modulus, which was the standard material. Then, he gripped another gelatin to answer whether it was harder or softer than the standard one. This process was repeated with each of the six different gelatins with recoding. If the operator answered that he cannot distinguish whether the gripped gelatin was harder or softer, the answer was recorded as incorrect. In this experiment, the force gain $S_{f}$ was 10 and 20. The distance between the master manipulator and the slave manipulator was $1.5 \mathrm{~m}$. The operator operated the master manipulator by looking directly at the slave manipulator and not by viewing it through the monitor. Figure 9 shows the gripping of gelatin by the slave manipulator and the master operation handle.
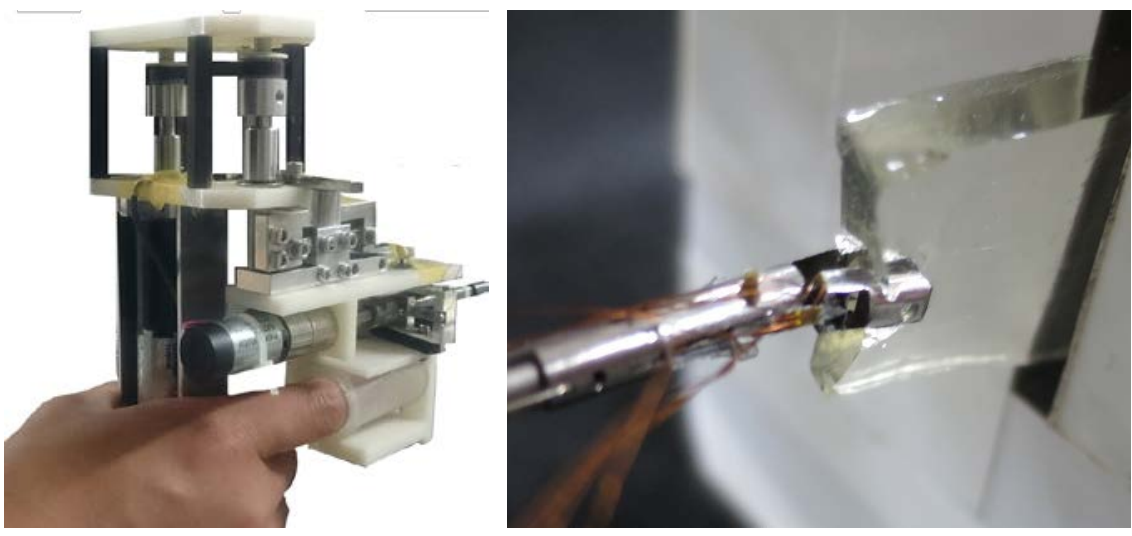

Fig. 9. Discrimination experiment to detect the target’s hardness.

Results

Figure 10 shows the plot of the total rate of the correct answers given by all the nine operators using each type of gelatin. The rate of correct answers decreased as the difference in the hardness values of the tested gelatins from that of the standard one decreased; however, this rate exceeded $60 \%$ at a force gain of 20, even when the difference in the hardness was $5 \mathrm{kPa}$. In this experiment, the maximum value of the actual gripping force at the slave manipulator was $0.1 \mathrm{~N}$. When the force gain was selected 20, the maximum gripping force at the master manipulator applied on the finger surface became $2 \mathrm{~N}$. The ability to distinguish the difference in the force at the index finger was excellent around force level 1 to $3 \mathrm{~N}$ throughout our experiment.

Figure 11 shows the correct-answer rate for each subject. The correct-answer rate at a force gain of 20 was higher than that at a force gain of 10 for all the operators. 


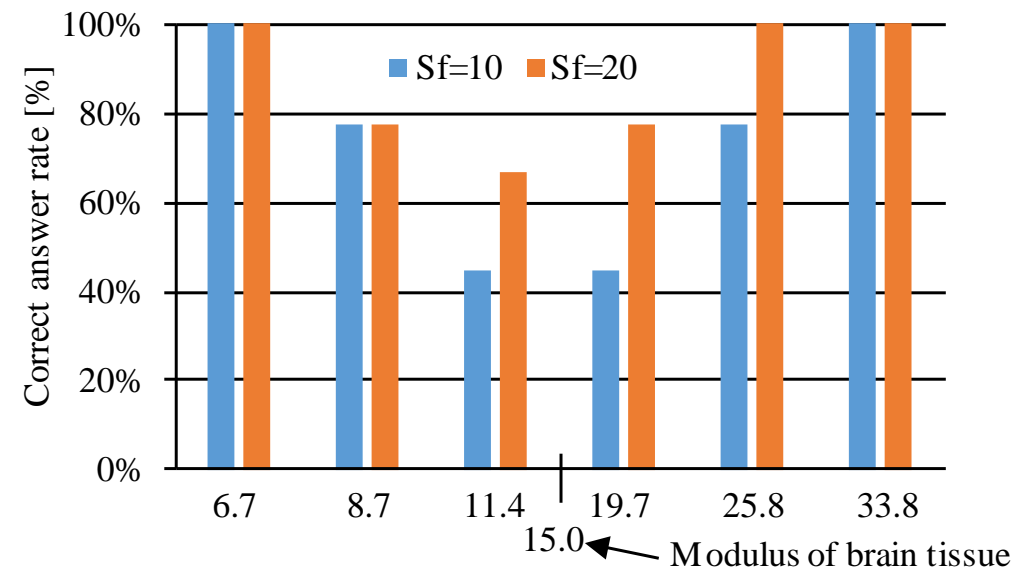

Young's modulus of gelatin $[\mathrm{kPa}]$

Fig. 10. Correct-answer rate for each gelatin.

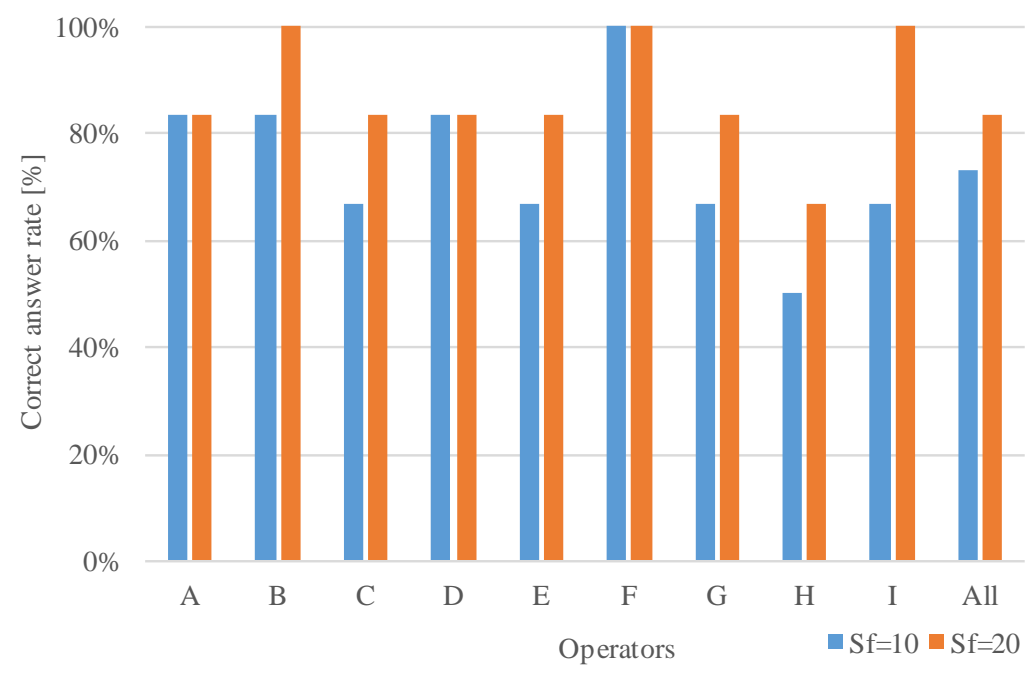

Fig. 11. Correct-answer rate for each operator.

These results indicate that the operator can distinguish the difference between the hardness of the tumor and that of normal tissue by using this gripping-force-feedback operating manipulator.

\section{Experiment to determine the difference in the pulling force}

An experiment was conducted to determine whether the operator can distinguish the difference in the pulling force in the event that the slave gripper pulls the soft material. In this experiment, two silicon materials with different adhering strengths to the base plate were used. The pulling force under the low-adherence condition (Low $\mathrm{P}_{\mathrm{f}}$ ) was $0.064 \mathrm{~N}$ and that under the high-adherence condition (High $\mathrm{P}_{\mathrm{f}}$ ) was $0.41 \mathrm{~N}$.

\section{Experimental procedure}

Two medical surgeons specializing in brain tumor resection conducted this experiment as operators. Fourteen silicon rubber pieces with a low or high adherence force were set on the plate. The operator gripped the silicon piece by observing the slave manipulator through the monitor. Then, three types of experiments were conducted. In the first type, the operator looked at the monitor and pulled the rubber piece with pulling-force feedback; this method is called "vision and force.” In the second type, the operator closed his eyes while pulling the rubber piece with pulling- 
force feedback; this method is called "force only." In the third type, the operator looked at the monitor and pulled the rubber piece with no pulling-force feedback; this method is called "vision only." In each case, if the operator felt that the pulling force was low, he pulled the gripper to confirm the resection. Moreover, if the operator felt that the pulling force was high, he stopped pulling the rubber. When he pulled off the rubber piece with low adhesion or kept the piece with high adhesion, the result was recorded as correct; however, if he did not pull out the piece with low adhesion and pulled out the piece with high adhesion, the result was recorded as wrong. The force gain was set to 20. Figure 12 shows the experimental set-up.



Fig. 12. Experimental set-up.

Experimental results

Figure 13 shows the correct-answer rate for each operator. If the rubber had high adhesion, the correct-answer rate was the lowest for the operators in the "vision only" experiment. The correctanswer rate in the "force only" experiment was higher than that in the "vision only" experiment; moreover, the correct-answer rate in the "vision and force" experiment was the highest. On the other hand, if the rubber had low adhesion, the correct-answer rate was low for the operators in the "force only" experiment.

With low-adhesion rubber, it was easy to distinguish whether the rubber can be detached or not by looking at the monitor because it was immediately detached by the low pulling force. Therefore, the correct-answer rate when the "vision only" method was used. With high-adhesion rubber, the operator could not distinguish whether the rubber can be pulled out when using the "vision only" method. Only in the "force only" method, he was able to distinguish whether it is hard to pull out the rubber using the large resistant load. Using the "vision and force" method, he could efficiently distinguish the adhesion force of the rubber.

Figure 14 shows the time taken to distinguish whether the adhesion is high or low in each experiment with standard deviation. These values are the time taken for giving the correct answer and were calculated from the time the operator started the pulling action to the time he completed the process by pulling out the rubber or by stopping the pulling action.

The time taken in the "vision and force" method was the minimum.

These results show that if the pulling force is large when attaching blood pipe or nerves, the "vision and force" method can be very useful to avoid the breaking of the blood pipes or damaging of nerves due to the application of excessive pulling force. It can also be helpful when a operator is unable to see the gripped tissue behind some other tissues as he can estimate the pulling hardness by using pulling-force feedback. 


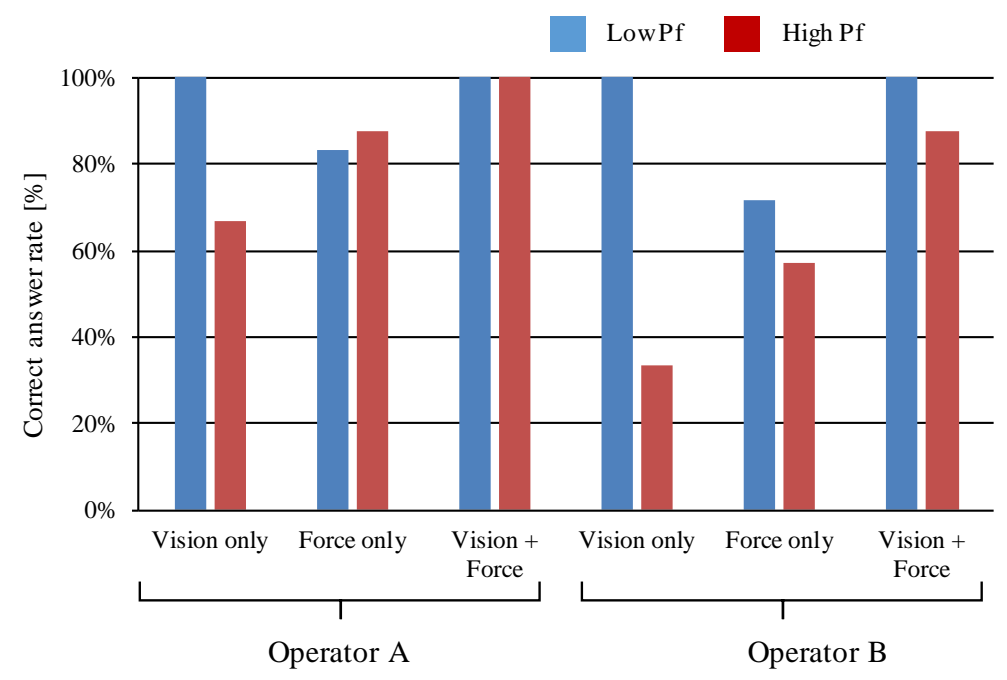

Fig. 13. Result of the experiment.

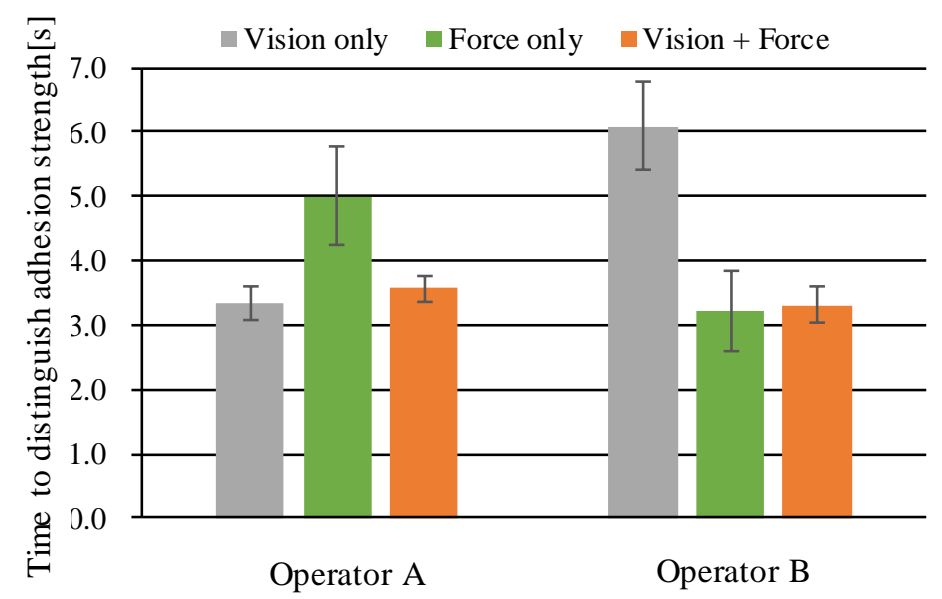

Fig. 14. Time taken to distinguish the adhesion strength.

\section{Discussion}

The most attractive characteristic of our finger-attachment device is that gripping force is reflected on the finger surface and pulling force is reflected on the side surface of the finger. Based on the experiments conducted to distinguish the hardness and pulling force of the gripped material, the usefulness of our finger-attachment device can be described as follows.

Based on the gripping-force feedback received on this attachment device, an operator can determine the hardness of brain tissue via the action of gripping; moreover, he can compare the hardness of normal brain tissue and a tumor. Because most of the tumor tissues are harder than normal tissue, he can confirm or estimate the area of a tumor. It may help in achieving precise resection of the tumor region. The navigation system used in nuclear magnetic resonance imaging or photodynamic diagnosis using 5-aminolevulinic acid (5-ALA) are useful for distinguishing the tumor region on the basis of vision images, and the force information at the surgical gripper will help in more precise resection of brain tumor.

Moreover, using pulling-force feedback, an operator can detect the resistance required to pull out a tumor. If he feels that the resistance to pull out the tumor is high, he may check whether there are other organs, e.g., blood tubes, attached to the gripper or entangled with the tumor material. It may prevent bleeding during tumor resection. 
If the region to be pulled off is hidden by other organs, force feedback may be help in the resection of the tumor. An operator can estimate the tumor region and determine whether it needs to be pulled out from the gripped material.

The advantage of our finger-attachment device is that the pulling force is independently applied on the operator's finger; a pulling force that can be felt on the finger is not influenced by the application of the gripping force, and the operator can feel the pulling-force resistance even if he applies excessive gripping force.

Although the master-slave manipulator system developed in this study is a prototype of the futuristic neurosurgery robot systems, sensing of gripping- and pulling-force feedback from force detection at the gripper to the master manipulator by the finger-attachment device are more important for precise resection of brain tumor without causing any damage to other organs.

The related research conducted thus far has only addressed the use of the test equipment in a laboratory. For realizing the application of the test equipment to real-life surgery, development of system reliability, operation, and handling performance is necessary. To this end, a larger number of operational tests should be conducted by operators to test the gripping and pulling of various materials under different conditions.

The design of the attachment system for force feedback should also be discussed. Human interaction involves several force-sensing activities. Pulling forces may be felt during the motion of the handling pole of the master manipulator. In our system, the pulling force can be fed back to the resistance of the handling pole. The actual gripping force and the pulling force in the tumor resection emerged on the surface of the slave gripper; thus, we intended to transfer those forces directly to the gripper and finger surface of the master manipulator. The sensory performance of the forefinger is very high. Therefore, feedback of the gripping force and pulling force onto the finger attachment may be an effective force feedback method; however, there must be some discussion on why the forefinger is stretched. The master handling manipulator resembles the activity of holding forceps using the thumb and forefinger. In this case, the forefinger is slightly stretched and the force is reflected onto the forefinger surface. The operation of the master manipulator resembles the activity of holding and operating forceps.

Several approaches have been used to realize haptic feedback systems, e.g., vibrotactile force perception [30]; however, these approaches suffer from a lack of quantitative friction force feedback on the fingertip. Our novel finger attachment system for friction feedback may be useful for operations involving friction.

\section{Conclusion}

Based on the basic test conducted to measure the performance of the index finger in detecting the pulling force under gripping-force application, it was found that the application of the pulling force on the side surface of the finger is more effective in distinguishing the pulling force when the gripping force is applied on the finger surface compared with the scenario when both the gripping and pulling forces are applied on the same finger-touching surface. Based on this knowledge, the finger-attachment device, which applies a gripping force on the finger surface and a pulling force on the finger's side surface, was developed. Based on the discrimination test performed to distinguish the hardness of the gripped material, an operator can distinguish whether the gripped material is harder or softer than normal brain tissue. This may help him to confirm whether the gripped material is a tumor. Based on the test conducted to distinguish the pulling force, an operator can distinguish the pulling-force resistance when he tries to pull off the soft material. Pulling-force feedback may help prevent the breaking of blood pipes when the material is trapped in the gripper or attached to the gripped tissue.

This gripping- and pulling-force feedback system developed herein for brain tumor resection will play an important role in the development of future neurosurgery robotic system.

All the experiments in this study were approved and performed in accordance with the ethical standards, and all the subjects in this study had provided informed consent before the experiment.

Conflict of interest: There is no conflict of interest in this study 


\section{References}

1. Cuschieri A (1995) Whither minimal access surgery: tribulations and expectations. Am J Surg 169:9-19. doi: 10.1016/S0002-9610(99)80104-4

2. Moreno-Egea A, Torralba JA, Morales G, Fernandez T, Guzman P, Hita G, Girela E, Corral M, Campillo A, Aguayo JL (2005) Laparoscopic repair of secondary lumbar hernias: open vs. laparoscopic surgery. A prospective, nonrandomized study. Cir Esp 77:159-62. doi: 10.1016/S0009-739X(05)70828-9

3. Chiu LH, Chen CH, Tu PC, Chang CW, Yen YK, Liu WM (2015) Comparison of robotic surgery and laparoscopy to perform total hysterectomy with pelvic adhesions or large uterus. J Minim Access Surg 11:87. doi: 10.4103/0972-9941.147718

4. Nguan C, Girvan A, Luke PP (2008) Robotic surgery versus laparoscopy; A comparison between two robotic systems and laparoscopy. J Robot Surg 1:263-268. doi: 10.1007/s11701007-0050-x

5. Bodner J, Augustin F, Wykypiel H, Fish J, Muehlmann G, Wetscher G, Schmid T (2005) The da Vinci robotic system for general surgical applications: a critical interim appraisal. Swiss Med Wkly 135:674-678. doi:10.4414/smw.2005.11022

6. Lanfranco AR, Castellanos AE, Desai JP, Meyers WC (2004) Robotic Surgery. Ann Surg 239:14-21. doi: 10.1097/01.sla.0000103020.19595.7d

7. Herron DM, Marohn M, Group TS-MRSC (2008) A consensus document on robotic surgery. Surg Endosc 22:313-325. doi: 10.1007/s00464-007-9727-5

8. Doulgeris JJ, Gonzalez-Blohm SA, Filis AK, Shea TM, Aghayev K, Vrionis FD (2015) Robotics in neurosurgery: evolution, current challenges, and compromises. Cancer Control 22:352-359.

9. Sutherland GR, Wolfsberger S, Lama S, Zarei-Nia K (2013) The evolution of neuroArm. Neurosurgery 72:27-32. doi: 10.1227/NEU.0b013e318270da19

10. Sutherland GR, Lama S, Gan LS, Wolfsberger S, Zareinia K (2013) Merging machines with microsurgery: clinical experience with neuroArm. J Neurosurg 118:521-9. doi: 10.3171/2012.11.JNS12877

11. Goto T, Miyahara T, Toyoda K, Okamoto J, Kakizawa Y, Koyama J, Fujiie MG, Hongo K (2009) Telesurgery of microscopic micromanipulator system "NeuRobot" in neurosurgery: interhospital preliminary study. J Brain Dis 1:45-53.

12. Goto T, Hongo K, Kakizawa Y, Muraoka H, Miyairi Y, Tanaka Y, Kobayashi S (2003) Clinical application of robotic telemanipulation system in neurosurgery. Case report. J Neurosurg 99:1082-1084. doi: 10.3171/jns.2003.99.6.1082

13. Mitsuishi M, Morita A, Sugita N, Sora S, Mochizuki R, Tanimoto K, Baek YM, Takahashi H, Harada K (2013) Master-slave robotic platform and its feasibility study for microneurosurgery. Int J Med Robot Comput Assist Surg 9:180-189. doi: 10.1002/rcs.1434

14. Arata J, Tada Y, Kozuka H, Wada T, Saito Y, Ikedo N, Hayashi Y, Fujii M, Kajita Y, Mizuno M, Wakabayashi T, Yoshida J, Fujimoto H (2011) Neurosurgical robotic system for brain tumor removal. Int J Comput Assist Radiol Surg 6:375-385. doi: 10.1007/s11548-010-0514-8 
15. Morita A, Sora S, Mitsuishi M, Warisawa S, Suruman K, Asai D, Arata J, Baba S, Takahashi H, Mochizuki R, Kirino T (2005) Microsurgical robotic system for the deep surgical field: development of a prototype and feasibility studies in animal and cadaveric models. $\mathrm{J}$ Neurosurg 103:320-327. doi: 10.3171/jns.2005.103.2.0320

16. Okayasu H, Okamoto J, Fujie MG, Umezu M, Iseki H (2003) Development of a hydraulicdriven flexible manipulator for neurosurgery. Int Congr Ser 1256:607-612. doi: 10.1016/S0531-5131(03)00324-8

17. Okamura AM (2009) Haptic Feedback in Robot-Assisted Minimally Invasive Surgery. Curr Opin Urol 19:102-107. doi: 10.1097/MOU.0b013e32831a478c.Haptic

18. Alleblas CCJ, Vleugels MPH, Nieboer TE (2016) Ergonomics of laparoscopic graspers and the importance of haptic feedback: the surgeons’ perspective. Gynecol Surg 13:379-384. doi: 10.1007/s10397-016-0959-z

19. Tholey G, Desai JP, Castellanos AE (2005) Force feedback plays a significant role in minimally invasive surgery: results and analysis. Ann Surg 241:102-9. doi: 10.1097/01.sla.0000149301.60553.1e

20. Wagner CR, Stylopoulos N, Howe RD (2002) The role of force feedback in surgery: analysis of blunt dissection. Proc - 10th Symp Haptic Interfaces Virtual Environ Teleoperator Syst HAPTICS 2002 68-74. doi: 10.1109/HAPTIC.2002.998943

21. MacFarlane M, Rosen J, Hannaford B, Pellegrini C, Sinanan M (1999) Force-feedback grasper helps restore sense of touch in minimally invasive surgery. J Gastrointest Surg 3:278-85.

22. Yoneyama T, Watanabe T, Kagawa H, Hamada J, Hayashi Y, Nakada M (2011) Force detecting gripper and flexible micro manipulator for neurosurgery. In: 2011 Annu. Int. Conf. IEEE Eng. Med. Biol. Soc. IEEE, pp 6695-6699.

23. Yoneyama T, Watanabe T, Kagawa H, Hamada J, Hayashi Y, Nakada M (2013) Forcedetecting gripper and force feedback system for neurosurgery applications. Int J Comput Assist Radiol Surg 8:819-829. doi: 10.1007/s11548-012-0807-1

24. Dehaene S (2003) The neural basis of the Weber-Fechner law: a logarithmic mental number line. Trends Cogn Sci 7:145-147. doi: 10.1016/S1364-6613(03)00055-X

25. Kanada Y, Yoneyama T, Watanabe T, Kagawa H, Sugiyama N, Tanaka K, Hanyu T (2013) Force feedback manipulating system for neurosurgery. Procedia CIRP 5:133-136. doi: 10.1016/j.procir.2013.01.027

26. Fujihira Y, Hanyu T, Kanada Y, Yoneyama T, Watanabe T, Kagawa H (2014) Gripping force feedback system for neurosurgery. Int $\mathrm{J}$ Autom Technol 8:83-94. doi: 10.20965/ijat.2014.p0083

27. Chinbe H, Yoneyama T, Watanabe T, Nakada M (2016) Haptic threshold for pulling force feedback on surgeon's fingertip in medical robotic systems. In: IECON 2016 - 42nd Annu. Conf. IEEE Ind. Electron. Soc. IEEE, pp 767-772.

28. Soza G, Grosso R, Nimsky C, Hastreiter P, Fahlbusch R, Greiner G (2005) Determination of the elasticity parameters of brain tissue with combined simulation and registration. Int $\mathrm{J}$ Med Robot 1:87-95. doi: 10.1002/rcs.32 
29. Kruse SA, Rose GH, Glaser KJ, Manduca A, Felmlee JP, Jack CR, Ehman RL (2008) Magnetic resonance elastography of the brain. Neuroimage 39:231-237. doi: 10.1016/j.neuroimage.2007.08.030

30. Hatzfeld C, Werthschütxky R (2010) Vibrotactile force perception thresholds at the fingertip, haptics: generating and perceiving tangible sensations, pp.99-104 doi: 10.1007/978-3-642-14064-8_15 